Pure and Applied Mathematics Quarterly

Volume 1, Number 1, 227-239, 2005

\title{
Weyl Chambers and Standard Monomial Theory for Poset Lattice Cones
}

\author{
Roger Howe
}

By a lattice cone, we mean the intersection with $\mathbf{Z}^{n}$ of a convex polyhedral cone in $\mathbf{R}^{n}$. It is a semigroup under vector addition. If the faces of the cone are defined by rational linear functionals, the semigroup will be finitely generated. The associated semigroup algebra is then a finitely generated, hence Noetherian, commutative algebra $[\mathrm{BH}]$. The varieties associated to these algebras are affine toric varieties $[\mathrm{Fu}]$.

The semigroup algebras of lattice cones appear naturally in invariant theory, as the algebras of invariants for tori (connected subgroups of the diagonal matrices, aka connected commutative reductive algebraic groups) acting on vector spaces. More recently, they have been playing a subtler role, as "model algebras" (technically, flat eformations) for various algebras such as the homogeneous coordinate rings of flag varieties, which have been the object of intensive study, under the rubric of "standard monomial theory." [GL], [Cal ], [Chi], [Hod], [HP].

This note describes a natural class of lattice cones attached to partially ordered sets. They include the cones which are used to describe standard monomial theory for classical groups. Their associated semigroup algebras are known as Hibi algebras [Hi]. These lattice cones have a natural decomposition into integrally simplicial cones. (In fact, these cones are Weyl chambers for the hyperoctahedral reflection group. They are all congruent to each other under this group.) This provides a description of the semigroup algebra as a sum of polynomial rings. It is this decomposition which constitutes/lies behind the classical standard monomial theory. It may be regarded as a standard monomial theory for Hibi algebras. This is an example of the viewpoint, that regarding a Hibi algebra as the semigroup ring of the lattice cone of a poset provides a simple approach to these rings. We show that having such a pleasant simplicial decomposition in fact characterizes this class of cones.

Received September 12, 2004. 
The bulk of this note is quite elementary, and it is hoped that it can serve as an introduction to this circle of ideas. The characterization theorem (Theorem 2.3) has the best chance of being novel.

This paper combines a number of topics which concerned Armand Borel during his productive career. It is a pleasure to dedicate it to his memory.

\section{Total orders and Weyl Chambers}

Let $X$ be a finite set, with \#(X) denoting its cardinality. Let $\mathbf{R}^{X}$ be the vector space of real-valued functions on $X$. Let $\mathbf{R}_{o}^{X}$ denote the open dense set of functions which are one-to-one. Since $\mathbf{R}$ is totally ordered, a given function $f \in \mathbf{R}_{o}^{X}$ implicitly defines a total ordering $>_{f}$ on $X$ :

$$
x>_{f} y \quad \Leftrightarrow \quad f(x)>f(y), \text { for } \quad x, y \in X .
$$

Given $f$ and $f^{\prime}$ in $\mathbf{R}_{o}^{X}$, we say that $f$ and $f^{\prime}$ are order equivalent if the orderings $>_{f}$ and $>_{f^{\prime}}$ are the same. Given $f \in \mathbf{R}_{o}^{X}$, let $C(f)$ denote the set of all $f^{\prime}$ in $\mathbf{R}_{o}^{X}$ which are order equivalent to $f$ :

$$
C(f)=\left\{f^{\prime} \in \mathbf{R}_{o}^{X}:>_{f^{\prime}}=>_{f}\right\} .
$$

1.3. Proposition: i) For any $f \in \mathbf{R}_{o}^{X}$, the set $C(f)$ is an open, convex cone. That is, if $f^{\prime} \in C(F)$, then $s f^{\prime} \in C(f)$ for any positive scalar $s$; and if $f_{1}$ and $f_{2}$ both belong to $C(f)$, then so does $f_{1}+f_{2}$. Also, $C(f)$ is an open set. ii) The sets $C(f)$ are exactly the connected components of $\mathbf{R}_{o}^{X}$. iii) The sets $C(f)$ are invariant under translation by constant functions. iv) The sets $C(f)$ are in natural one-to-one correspondence with the total orderings on $X$. v) The sets $C(f)$ are permuted simply transitively by the group $S_{X}$ of all permutations of $X$.

Proof: The assertions i) and iii) are very simple to check. For ii), consider two functions $f_{0}$ and $f_{1}$ in $C\left(f\right.$. Then $t \rightarrow t f_{0}+(1-t) f_{1}$ for $0 \leq t \leq 1$, defines a continuous curve connecting $f_{0}$ with $f_{1}$, and it belongs to $C(F)$ by part i). On the other hand, if $C\left(f_{0}\right) \neq C\left(f_{1}\right)$, then for some $x$ and $y$ in $X$, we must have $f_{0}(x)>f_{0}(y)$, but $f_{1}(x)<f_{1}(y)$. Hence, if $f_{t}$ is a continuous family of functions conecting $f_{0}$ and $f_{1}$, we must have $f_{t}(x)-f_{t}(y)=0$ for some $t$ between 0 and 1 by 
the Intermediate Value Theorem. Thus, $f_{0}$ and $f_{1}$ are not in the same component of $\mathbf{R}_{o}^{X}$.

For iv), let $>_{o}$ be any total ordering on $X$. Define $f_{>_{o}}$ by the formula $f_{>_{o}}(x)=$ $\#\left(\left\{y \in X: x>_{o} y\right\}+1\right.$. Then $f_{>_{o}}$ is a one-to-one function on $X$ to the set $\{1,2,3, \ldots, \#(X)\} \subset \mathbf{Z} \subset \mathbf{R}$. It is sraightforward to check that $>_{f_{>_{0}}}=>_{o}$. Finally, assertion v) simply amounts to the observation that, given two total orderings $>$ and $>^{\prime}$ on $X$, there is a unique permutation $\sigma$ of $X$, and such that $>^{\prime}=>\circ \sigma$.

Let $\mathbf{R}^{+X} \subset \mathbf{R}^{X}$ be the cone of non-negative valued functions on $X$. Given any $f$ in $\mathbf{R}^{X}$, if we add a sufficiently large positive constant to $f$, we will get a function with non-negative values. From property iii) of Proposition 1.3, we see that each cone $C(f)$ intersects $\mathbf{R}^{+X}$ non-trivially. Set

$$
C(f) \cap \mathbf{R}^{+X}=C^{+}(f) .
$$

We have a parallel statement to Proposition 1.3 for the $C^{+}(f)$. We set $\mathbf{R}_{o}^{+X}=$ $\mathbf{R}^{+X} \cap \mathbf{R}_{o}^{X}$.

1.3 bis. Proposition: i) For any $f \in \mathbf{R}^{+}{ }_{o}^{X}$, the set $C^{+}(f)$ is an open, convex cone. That is, if $f^{\prime} \in C^{+}(F)$, then $s f^{\prime} \in C^{+}(f)$ for any positive scalar $s$; and if $f_{1}$ and $f_{2}$ both belong to $C^{+}(f)$, then so does $f_{1}+f_{2}$. Also, $C^{+}(f)$ is an open set. ii) The sets $C^{+}(f)$ are exactly the connected components of $\mathbf{R}_{o}^{+X}$. iii) The sets $C^{+}(f)$ are taken into themselves under translation by non-negative constant functions. iv) The sets $C^{+}(f)$ are in natural one-to-one correspondence with the total orderings on $X$. v) The sets $C^{+}(f)$ are permuted simply transitively by the group $S_{X}$ of all permutations of $X$.

Let $\bar{C}^{+}(F)$ denote the closure of $C^{+}(f)$. This is a closed convex subcone of $\mathbf{R}^{+X}$. Here is a careful description of $\bar{C}^{+}(F)$.

Recall that a simplicial cone is the collection of non-negative linear combinations of a linearly independent set $B$ of vectors. The vectors in $B$ are called the generators of the cone. They are determined up to non-negative multiples.

Recall also that a subset $Y$ of a partially ordered set $X$ is called increasing (aka order ideal [St]) provided that, for any $y \in Y$ and any $y^{\prime} \in X$ with $y^{\prime} \geq y$, we also have $y^{\prime} \in Y$.

The function $f$ in $\mathbf{R}_{o}^{+X}$ defines the total order $>_{f}$ on $X$. For $x \in X$, set

$$
\left(f^{-1}\right)^{+}(x)=\left\{y \in X: y \geq_{f} \quad x\right\}=\{y \in X: f(y) \geq f(x)\} .
$$


This is clearly an increasing set in $X$ with respect to the order $>_{f}$. It is easy to show that, since $>_{f}$ is a total ordering, the sets (1.4) are all the increasing subsets of $X$ with respect to $>_{f}$.

For each $x$ in $X$, there is a unique $y$ in $X$ which is the "next larger" with respect to $>_{f}$. That is, $y>_{f} x$, and there is no $z$ in $X$ such that $y>_{f} z>_{f} x$. We will denote this element $y$ by $x+_{f} 1$. We will also write $x=y-{ }_{f} 1$. Let $x_{o_{f}}$ denote the minimum element in $X$ with respect to the order $>_{f}$.

1.5. Proposition. i) The cone $\bar{C}^{+}(F)$ is simplicial. More precisely, it is the non-negative span of the characteristic functions of increasing subsets of $X$ with respect to the total order $>_{f}$. These are exactly the sets $\left(f^{-1}\right)^{+}(x)$ of definition (1.4).

ii) The cone $\bar{C}^{+}(f)$ is defined by the linear inequalities

$$
\bar{C}^{+}(f)=\left\{\phi \in \mathbf{R}^{+X}: \phi(y) \geq \phi(x) \Leftrightarrow f(y)>f(x)\right.
$$

and

$$
\phi(x) \geq 0\}=\left\{\phi \in \mathbf{R}^{+X}: \phi(y) \geq \phi\left(y-{ }_{f} 1\right) \geq 0\right\} .
$$

Proof: For $x$ in $X$, let $\chi_{\left(f^{-1}\right)^{+}(x)}=\chi^{+}(x)$ denote the characteristic function of the set $\left(f^{-1}\right)^{+}(x)$ of formula (1.4). Then $\chi^{+}\left(x+_{f} 1\right)-\chi^{+}(x)=\delta_{x}$ is the characteristic function of the one-element set $\{x\}$. Thus, for any function $\phi$ in $\mathbf{R}^{x}$, we may write

$$
\phi=\sum_{x \in X} \phi(x)\left(\chi^{+}(x)-\chi^{+}\left(x+{ }_{f} 1\right)\right)=\sum_{y \in X}\left(\phi(y)-\phi\left(y-{ }_{f} 1\right)\right) \chi^{+}(y) .
$$

In the second sum, we adopt the convention that if $y$ is the minimum element in $X$ with respect to $>_{f}$, then $\phi\left(x-{ }_{f} 1\right)=0$.

From formula (1.6), we can see that $\phi$ is a non-negative combination of the $\chi^{+}(x)$ exactly when $\phi(y) \geq \phi\left(y-{ }_{f} 1\right)$ for all $y$ in $X$. The condition $\phi(y)>\phi\left(y-{ }_{f} 1\right)$ is precisely the condition to belong to $C^{+}(f)$. It is obvious that weakening this condition from strict inequality to weak inequality will produce the closure of $C^{+}(f)$. This is the assertion ii) of the proposition. Further, the sum (1.6) exhibits $\bar{C}^{+}(f)$ as the non-negative combinations of the characteristic functions $\chi^{+}(x)$. This is the assertion i) of the proposition.

1.7. Remark: The sets $C(f)$ and $C^{+}(f)$ appear in the theory of reflection groups [BG], where they are known as Weyl chambers. The sets $C(f)$ are the 
(open) Weyl chambers for the action of the symmetric group $S_{X}$ on $\mathbf{R}^{X}$. The sets $\bar{C}^{+}(f)$ are the (closed) Weyl chambers for the "hyperoctohedral" group of permutations together with sign changes (multiplication by \pm 1 valued functions). From here on, we will refer to the cones $\bar{C}^{+}(f)$ as Weyl chambers.

\section{Posets and Cones}

Now suppose that the set $X$ is a poset; that is, $X$ is endowed with a partial order (which we will denote simply by $\geq$, and hope that no confusion will arise). To this structure, we can attach a canonical cone in $\mathbf{R}^{X}$. We will say that a function $f$ in $\mathbf{R}^{X}$ is compatible with $\geq$ provided that for a pair of elements $x$, $y$ in $X$, if $y \geq x$, then $f(x) \geq f(y)$. Also, we will say that a total ordering on $X$ is compatible with $\geq$ if whenever $y \geq x$, then also $y$ dominates $x$ with respect to the total ordering. Evidently, if $f$ belongs to $\mathbf{R}_{o}^{X}$, then $f$ is compatible with $\geq$ if and only if the associated total ordering $>_{f}$ is compatible with $\geq$. Let $\mathbf{R}^{X}(\geq)$ denote the set of all functions in $\mathbf{R}^{X}$ which are compatible with $X$, and let $\mathbf{R}^{+X}(\geq)=\mathbf{R}^{X}(\geq) \cap \mathbf{R}^{+X}$.

2.1. Proposition: i) The sets $\mathbf{R}^{X}(\geq)$ and $\mathbf{R}^{+X}(\geq)$ are closed convex cones. ii) The set $\mathbf{R}^{X}(\geq)$ is invariant under translations by constant functions. iii) The set $\mathbf{R}^{+X}(\geq)$ is a union of the Weyl chambers $\bar{C}^{+}(f)$ for $f$ in $\mathbf{R}_{o}^{+X}$ compatible with $\geq$.

Proof: i) is sraightforward to check. The closedness results from the fact that weak inqeualities are used to define compatibility. Since translation by constants does not affect the order of function values, statement ii) is also evident. Since the compatibility with $\geq$ of a function $f$ in $\mathbf{R}_{o}^{X}$ is equivalent with compatibility of the order $>_{f}$ with $\geq$, and since $>_{f}$ depends only on the (open) Weyl chamber to which $f$ belongs, it is clear that if an open Weyl chamber intersects $\mathbf{R}^{+X}(\geq)$, it is entirely contained inside it. Since $\mathbf{R}^{+X}(\geq)$ itself is closed, this means that the entire closed Weyl chamber will be contained in $\mathbf{R}^{+X}(\geq)$.

It remains to argue that these Weyl chambers contained in $\mathbf{R}^{+X}(\geq)$ fill it up entirely. Since we can find total orders compatible with $\geq, \mathbf{R}^{+X}(\geq)$ will contain some Weyl chambers, and so it has non-empty interior. This interior must intersect $\mathbf{R}_{o}^{+}{ }_{o}^{X}$ in a dense open subset. Thus, the union of the Weyl chambers contained in $\mathbf{R}^{+X}(\geq)$ is dense in $\mathbf{R}^{+X}(\geq)$, and since it is also closed, being a finite union of closed sets, it is the whole cone. 
2.2. Remark: The cones $\bar{C}^{+}(f)$ have disjoint interiors. They intersect only along proper subfaces. We will call such a union of cones almost disjoint. It is notable that the properties of the cones $\mathbf{R}^{+X}(\geq)$ listed in Proposition 2.1 in fact characterize such cones.

2.3. Theorem: Any closed convex cone in $\mathbf{R}^{+X}$ which is a union of Weyl chambers $\bar{C}^{+}(f)$ is of the form $\mathbf{R}^{+X}(\geq)$, for an appropriate partial ordering $\geq$ on $X$.

Proof: Each Weyl chamber $\bar{C}^{+}(f)$ corresponds to a total ordering $>_{f}$ on $X$. Given a collection $\omega$ of such total orders, let $\geq_{\omega}$ be the strongest partial ordering compatible with all the total orders in $\omega$. That is, for two (distinct) elements $x$ and $y$ in $X$, we have

$$
y \geq_{\omega} x \quad \Leftrightarrow \quad y>_{f} x, \text { for all }>_{f} \in \omega .
$$

If now we consider the cone $\mathbf{R}^{+X}\left(\geq_{\omega}\right)$, it is clear that $\bar{C}^{+}(f) \subset \mathbf{R}^{+X}\left(\geq_{\omega}\right)$, for any $f$ such that $>_{f}$ belongs to $\omega$. What we need to show is, that if $\omega$ is the collection of total orderings attached to Weyl chambers filling out a convex cone $C$, then we have $\mathbf{R}^{+X}\left(\geq_{\omega}\right)=C$.

Since $C$ is a union of Weyl chambers, it is a polyhedral cone. Thus, it will be the intersection of a finite number of half spaces, defined by its $(\#(X)-1)$ dimensional faces. Any such codimension 1 face of $C$ must also be a face of one of its constituent cones $\bar{C}^{+}(f)$. According to Proposition 1.5 ii), the half space $H$ defined by such a face is of the form

$$
H=H_{(y, x)}=\left\{f \in \mathbf{R}^{+X}: f(y) \geq f(x)\right\} .
$$

If $f$ belongs to $\mathbf{R}_{o}^{X}$, then none of its values are the same, so for such an $f$ in $H$ will satisfy $f(y)>f(x)$. This means that the corresponding total order $>_{f}$ will require that $y>_{f} x$. Since this is true for all one-to-one $f$ in $C$, this means that $y \geq_{\omega} x$. Thus, the inequality $f(x) \geq f(y)$ will hold in $\mathbf{R}^{+X}\left(\geq_{\omega}\right)$, or in other words, $\mathbf{R}^{+X}\left(\geq_{\omega}\right) \subseteq H_{(y, x)}$. Since this is true for any of the half spaces $H_{(y, x)}$ defining $C$, it follows that $\mathbf{R}^{+X}\left(\geq_{\omega}\right) \subseteq C$. Since the reveerse conclusion is automatically true, we conclude that $\mathbf{R}^{+X}\left(\geq_{\omega}\right)=C$, as desired. 


\section{Integral structure}

The group $\mathbf{Z}^{X}$ of integer valued functions on our set $X$ may be thought of as a lattice in the real vector space $\mathbf{R}^{X}$. The semigroup $\mathbf{Z}^{+X}$ of non-negative integer valued functions is then the intersection of $\mathbf{Z}^{X}$ with $\mathbf{R}^{+X}$ of non-negative valued functions. We want to study the lattice cone $\mathbf{Z}^{+X}(\geq)$ of non-negative integer valued functions on $X$, which are also compatible with the partial order $\geq$ on $X$.

The set $\mathbf{Z}^{+}$is a free semigroup. More precisely, it is generated by the standard basis vectors $\delta_{x}$. The function $\delta_{x}$ takes the value 0 everywhere except at the element $x$, where it takes the value 1 . Note that $\mathbf{Z}^{+X}=\mathbf{Z}^{X} \cap \mathbf{R}^{+X}$. The cone $\mathbf{R}^{+X}$ is a simplicial cone, and the $\delta_{x}$ are its generators. More precisely, $\delta_{x}$ is the smallest integer vector in the ray of $\mathbf{R}^{+X}$ that it generates. Clearly, the non-negative integer combinations of the $\delta_{x}$ form a subsemigroup of $\mathbf{Z}^{+X}$; in fact, this subsemigroup is all of $\mathbf{Z}^{+X}$. We thus call $\mathbf{R}^{+X}$ an integrally simplicial cone. More generally, let $C$ be a simplicial cone in $\mathbf{R}^{X}$, with generating set $B$. Suppose that $C$ is a rational cone, in the sense that each element of $B$ has a multiple which is integral (i.e., takes integer values). Without loss of generality, we may assume that the elements of $B$ are themselves integral, and further, that they are the smallest possible integral generators, meaning that no element $\frac{b}{k}$, for $b \in B$ and $k \in \mathbf{Z}$ is also integral. Then the $\mathbf{Z}^{+}$combinations of elements of $B$ will be a semigroup $\mathbf{Z}^{+} B$ which lies inside $C \cap \mathbf{Z}^{X}$. We will say that the cone $C$ is integrally simplicial if it happens that $\mathbf{Z}^{+} B=C \cap \mathbf{Z}^{X}$. In this case, the semigroup $C \cap \mathbf{Z}^{X}$ is free; and this is in fact eqivalent to $C$ being integrally simplicial.

3.1. Example: Consider the cone $C \subset \mathbf{R}^{2}$ defined by

$$
C=\left\{\left[\begin{array}{l}
x \\
y
\end{array}\right]: \frac{x}{2} \leq y \leq 2 x\right\} .
$$

It can be seen that $C$ is the simplicial cone generated by vectors $\left[\begin{array}{l}2 \\ 1\end{array}\right]$ and $\left[\begin{array}{l}1 \\ 2\end{array}\right]$. However, it is not integrally simplicial. since the points $\left[\begin{array}{l}1 \\ 1\end{array}\right]$ and $\left[\begin{array}{l}2 \\ 2\end{array}\right]$ belong to $C \cap \mathbf{Z}^{2}$, but are not non-negative integer combinations of the elements of the generators for $C$.

3.2. Proposition: The Weyl chambers $\bar{C}^{+}(f)$ are integrally simplicial cones. More precisely, the generators $\chi^{+}(x)$ defined in the proof of Proposition 1.5 define the canonical generators of $\bar{C}^{+}(f) \cap \mathbf{Z}^{X}$.

Proof: The fact that any element of $\bar{C}^{+}(f) \cap \mathbf{Z}^{X}$ belongs to the non-negative 
integer combinations of the $\chi_{x}$ follows from the formula (1.6).

We can now describe the lattice cone $\mathbf{Z}^{+}(\geq)$. Let $J^{*}(X, \geq)$ denote the set of increasing subsets of $X$, with respect to the partial order $\geq$. (This notation is compatible with the notation of [St]; there the collection of decreasing sets of $X$ is denoted $J(X)$. There decreasing subsets are called "order ideals", and increasing subsets are called "dual order ideals". Of course, $J(X, \geq)$ and $J^{*}(X, \geq)$ are in canonical bijection via set complementation. ) Note that $J^{*}(X, \geq)$ is ordered by set inclusion, and also, it is closed under the operations of union and intersection - it is a lattice of sets. Here "lattice" is used in its poset sense, rather than its geometric group theoretic sense, (as in "lattice cone"). For a set $A \subseteq X$, we let $\chi_{A}$ denote the characteristic function of $A$. We observe that the function $\chi_{A}$ is compatible with $\geq$ if and only if $A$ is an increasing subset of $X$.

Recall that a chain in a poset $X$ is a subset $Y$ which is totally ordered with respect to the partial order $\geq$ on $X$. It is well known that a maximal chain in $J^{*}(X, \geq)$ consists of $\#(X)$ sets. Such a chain gives rise to a total ordering on $X$ by declaring the sets of the maximal chain to be the increasing subsets for the corresponding total ordering. This correspondence is in fact a bijection between the set of total orderings on $X$ compatible with $\geq$ and the maximal chains in $J^{*}(X, \geq)$.

3.3. Theorem: i) The lattice cone $\mathbf{Z}^{+X}(\geq)$ is generated by the characteristic functions $\left\{\chi_{A} ; A \in J^{*}(X, \geq)\right.$. A defining set of relations among these generators is given by the equations

$$
\chi_{A}+\chi_{B}=\chi_{A \cup B}+\chi_{A \cap B} .
$$

ii) The lattice cone $\mathbf{Z}^{+X}(\geq)$ is the almost disjoint union of the integrally simplicial cones $\bar{C}^{+}(f) \cap \mathbf{Z}^{X}$, for $f$ in $\mathbf{R}_{o}^{+X}$. These cones are in natural bijection with the total orderings on $X$ compatible with $\geq$.

Proof: Statement ii) follows from Propositions 2.1 and 3.2. The description of the generators also follows from Proposition 3.2, since the generators of each cone $\bar{C}^{+}(f) \cap \mathbf{Z}^{X}$ are characteristic functions of increasing subsets of $X$. The relations (3.4) are obviously valid. Hence, the only part of the theorem that remains to be checked is the statement that the relations (3.4) suffice to define the addition in $\mathbf{Z}^{+X}(\geq)$.

Since $\mathbf{Z}^{+X}(\geq)$ is the union of the subcones $\bar{C}^{+}(f) \cap \mathbf{Z}^{X}$, any element in 
$\mathbf{Z}^{+}{ }^{X}(\geq)$ has a standard expression as a sum

$$
\sum_{j}^{\#(X)} c_{j} \chi_{A_{j}}
$$

where the $A_{j}$ are increasing subsets of $X$, with $A_{j} \subset A_{j+1}$, and $\#\left(A_{j}\right)=j$. In other words, the $A_{j}$ form a maximal chain in $J^{*}(X, \geq)$.

We will show by induction that the relations (3.4) allow one to rewrite any sum of generators in the form (3.5). For this, it will suffice to take a sum of type (3.5), add to it one characteristic function $\chi_{B}$, and show how to rewrite the result in the form (3.5) usng the relations (3.4).

Hence, we consider a sum $\chi_{B}+\sum_{j}^{\#(X)} c_{j} \chi_{A_{j}}$, and we want to reexpress it in the form (3.5). If it happens that $B=A_{m}$ for some $m$, then we may simply incorporate $\chi_{B}$ into the sum on the $\chi_{A_{j}}$ without further ado. So we may as well assume that $B$ is not one of the $A_{m}$. If $\#(X)=n$, then $A_{n}=X$, so we know that for sufficiently large $m, B \subset A_{m}$. Let $\ell$ be the largest index such that $A_{\ell} \not \supset B$. Then since $B \subset A_{\ell+1}$, we must have $A_{\ell} \cup B=A_{\ell+1}$. Thus, relation (3.4) implies that $\chi_{A_{\ell}}+\chi_{B}=\chi_{A_{\ell+1}}+\chi_{A_{e l l} \cap B}$. If $c_{\ell} \neq 0$, this allows us to write

$$
\chi_{B}+\sum_{j}^{\#(X)} c_{j} \chi_{A_{j}}=\chi_{B \cap A_{\ell}}+\sum_{j}^{\#(X)} c_{j}^{\prime} \chi_{A_{j}},
$$

where $c_{\ell+1}^{\prime}=c_{\ell+1}+1$, and $c_{\ell}^{\prime}=c_{\ell}-1$, and $c_{j}^{\prime}=c_{j}$ for the other $j$. Since $\#\left(B \cap A_{\ell}\right)$ is less than $\#(B)$, this allows us to conclude by induction that we can achieve the reduction we desire.

However, if $c_{\ell}=0$, then this would result in a sum with a negative coefficient. In this situation, we have to refine the above procedure slightly. Instead of working with $A_{\ell}$, we look for the largest index $\ell^{\prime}$ such that $A_{\ell^{\prime}} \not \supset B$ and $c_{\ell^{\prime}} \neq 0$. Since then $c_{j}=0$ for $\ell^{\prime}<j<\ell+1$, these sets $A_{j}$ are not actually involved in the sum $\sum_{j}^{\#(X)} c_{j} \chi_{A_{j}}$, which is therefore effectively a sum over a non-maximal chain in $J^{*}(X, \geq)$. We see then that if we delete these $A_{j}$ from the chain, and adjoin $A_{\ell^{\prime}} \cup B$ to it, we will again obtain a chain in $J^{*}(X, \geq)$. Now we may write

$$
\chi_{B}+\sum_{j}^{\#(X)} c_{j} \chi_{A_{j}}=\chi_{B \cap A_{\ell^{\prime}}}+\left(\chi_{A_{\ell^{\prime}} \cup B}+\sum_{j}^{\#(X)} c_{j}^{\prime} \chi_{A_{j}}\right) .
$$

In this sum, the set $B \cap A_{\ell^{\prime}}$ has smaller cardinality than $B$, and the sum in parentheses is in standard form: it is a sum over characteristic functions of a chain 
in $J^{*}(X, \geq)$. Again, by induction we see we can convert this sum to standard form using relations (3.4). This concludes the proof of Theorem 3.3.

3.4: The characteristic functions $\chi_{A}$ for increasing sets $A \subseteq X$ are clearly all needed to form the simplicial cones $C^{+}(f)$ contained in $\mathbf{Z}^{+}(\geq)$. They provide the extreme rays of these cones. Hence all extreme rays of the cone $\mathbf{Z}^{+}(\geq)$must be generated by the $\chi_{A}$. However, a given $\chi_{A}$ may or may not be extreme in the the cone $\mathbf{Z}^{+}(\geq)$. It is quite easy to give a criterion for this to be so. Proposition. i)

Given a subset $A \subseteq X$, the characteristic function is extreme in the cone $\mathbf{Z}^{+}(\geq)$ if and only if $A$ can not be written as a disjoint union $A=A_{1} \cup A_{2}$ of increasing subsets.

ii) Hence, all functions $\chi_{A}$ of increasing subsets fo $X$ define extreme rays of $\mathbf{Z}^{+}(\geq)$if and only if $X$ has a unique maximum element.

Proof. Suppose that $\chi_{A}=\sum_{j} c_{j} \chi_{A_{j}}$ is an expression for $\chi_{A}$ as a positive linear combination of characteristic functions of other increasing sets. Since all terms are non-negative, it is clear that every $A_{j} \subseteq A$. In order for the sum for $\chi_{A}$ to be non-trivial, we should have $A_{j_{o}} \neq A$ for some $j_{o}$. Consider the sum with the term $c_{j_{o}} \chi_{A_{j_{o}}}$ omitted. This will take two values, 1 and $1-c_{j_{o}}$. The set where it takes the value 1 is increasing, since the sum is in the cone $\mathbf{Z}^{+}(\geq)$, and the set where it takes the value $1-c_{j_{o}}$ is $A_{j_{o}}$, which is also increasing. These sets are disjoint and together they cover $A$, so $A$ is a disjoint union of increasing sets, as claimed. The converse is clear. This proves statement i).

For statement ii), suppose that $A$ does not have a unique maximum element. Let $x$ and $y$ be two maximal elements. Then $A=\{x, y\}=\{x\} \cup\{y\}$ is a union of two increasing subsets. Conversely, if $X$ has a unique maximum element, then it will belong to all increasing sets, and there can be no pair of disjoint increasing sets.

\section{Standard monomial theory for Hibi algebras}

We now translate the discussion above into commutative algebra. We turn our attention to the semigroup ring $\mathbf{C}\left(\mathbf{Z}^{+X}(\geq)\right)$, which consists of formal linear combinations of elements of $\mathbf{Z}^{+X}(\geq)$, with multiplication extended linearly from the semigroup law in $\mathbf{Z}^{+X}(\geq)$. What was regarded as addition in $\mathbf{Z}^{+X}(\geq)$ now gets called multiplication in $\mathbf{C}\left(\mathbf{Z}^{+X}(\geq)\right)$. To bridge this change of point of view, we will use the following notation. Given an element of $\mathbf{Z}^{+X}(\geq)$, when we think of it as an element of $\mathbf{C}\left(\mathbf{Z}^{+}(\geq)\right)$, we will surround it with square brackets: thus an element $b$ of $\mathbf{Z}^{+X}(\geq)$ corresponds to an element $[b]$ of $\mathbf{C}\left(\mathbf{Z}^{+X}(\geq)\right)$. The 
multiplication in $\mathbf{C}\left(\mathbf{Z}^{+}{ }^{X}(\geq)\right)$ is then defined by the rule

$$
[b]\left[b^{\prime}\right]=\left[b+b^{\prime}\right] .
$$

The algebra $\mathbf{C}\left(\mathbf{Z}^{+X}(\geq)\right)$ is generated as an algebra by any set of generators for the semigroup $\mathbf{Z}^{+X}(\geq)$. Theorem 3.3 tells us that we may think of $\mathbf{C}\left(\mathbf{Z}^{+X}(\geq)\right)$ as being generated by the set $J^{*}(X, \geq)$. For a given element $A$ of $J^{*}(X, \geq)$, the corresponding element of $\mathbf{C}\left(\mathbf{Z}^{+X}(\geq)\right)$ is $\left[\chi_{A}\right]$. We will abbreviate this to simply $[A]$.

As we have noted, the set $J^{*}(X, \geq)$ is a lattice of subsets of $X$. In particlar, it has the operations of intersection and union, which make it into a distributive lattice in the sense of partially ordered sets. In this context, the relations (3.4) may be rewritten as

$$
[A][B]=[A \cup B][A \cap B] .
$$

These are the relations of the Hibi ring [Hi] attached to the distributive lattice $J^{*}(X, \geq)$. In other words, the semigroup $\operatorname{ring} \mathbf{C}\left(\mathbf{Z}^{+X}(\geq)\right)$ is a Hibi ring. Conversely, every Hibi ring is a $\mathbf{C}\left(\mathbf{Z}^{+}{ }^{X}(\geq)\right)$ for some partial order. In the light of the discussion below, Theorem 2.3 may be thought of as characterizing these rings as having a particularly nice standard monomial theory.

The decomposition of $\mathbf{Z}^{+X}(\geq)$ into the integrally simplicial subcones $\bar{C}^{+}(f)$ translates into the context of $\mathbf{C}\left(\mathbf{Z}^{+X}(\geq)\right)$ as follows. Since each semigroup $\bar{C}^{+}(f)$ is free, the corresponding semigroup $\operatorname{ring} \mathbf{C}\left(\bar{C}^{+}(f)\right)$ is a polynomial ring. The fact that $\mathbf{Z}^{+X}(\geq)$ is the union of the $\bar{C}^{+}(f)$ means that the $\operatorname{ring} \mathbf{C}\left(\mathbf{Z}^{+}{ }^{X}(\geq)\right)$ is the sum of the polynomial rings $\mathbf{C}\left(\bar{C}^{+}(f)\right)$. The sum is not quite direct, but in some sense it does not fail badly to be direct. The intersection of any two Weyl chambers, $\bar{C}^{+}(f) \cap \bar{C}^{+}\left(f^{\prime}\right)$, is an integrally sumplicial subcone generated by the intersection of the sets of generators for the individual Weyl chambers. This translates to the statement that the two polynomial rings $\mathbf{C}\left(\bar{C}^{+}(f)\right)$ and $\mathbf{C}\left(\bar{C}^{+}\left(f^{\prime}\right)\right)$ intersect in a polynomial ring of lower dimension (and with generators equal to the intersection of the given sets of generators). We will express this circumstance by saying that $\mathbf{C}\left(\mathbf{Z}^{+}{ }^{X}(\geq)\right)$ is the almost direct sum of the polynomial rings $\mathbf{C}\left(\bar{C}^{+}(f)\right)$.

We summarize this discussion with the following statement.

4.3. Theorem. i) The semigroup ring $\mathbf{C}\left(\mathbf{Z}^{+X}(\geq)\right)$ is the Hibi ring attached to the distributive lattice $J^{*}(X, \geq)$. It is generated by the elements of $J^{*}(X, \geq)$, subject to the relations (4.2). 
ii) $\mathbf{C}\left(\mathbf{Z}^{+}{ }^{X}(\geq)\right)$ is the almost direct sum of the subalgebras generated by maximal chains in $J^{*}(X, \geq)$, which are polynomial rings. These are in one-to-one correspondence with the total orderings on $X$ compatible with $\geq$. In other words, the monomials in elements of maximal chains in $J^{*}(X, \geq)$ (aka standard monomials), form a basis for $\mathbf{C}\left(\mathbf{Z}^{+}(\geq)\right)$.

4.4. Remark: The main result of the original standard monomial theory of Hodge [Hod]. [HP] for the coordinate ring of the flag manifold for $G L_{n}$ was a description of this ring as an almost direct sum of polynomial rings. The generating sets of these polynomial rings in fact corresponded to maximal chains in a certain distributive lattice, and thereby to the total orderings on an associated poset, via the fundamental theorem of distributive lattices [St]. The poset GT in question is the one involved in defining Gelfand-Tsetlin patterns [GT], [Pr]. It is a piece of $\mathbf{Z}^{+2}$ with its standard coordinatewise order. However, these connections were not made until much later. The connection was made more salient (but apparently still implicit) by the demonstration in [GL] that the coordinate ring of the flag manifold for $G L_{n}$ is in fact a flat deformation of the semigroup ring $\mathbf{C}\left(\mathbf{Z}^{+G T}(\geq)\right)$. In view of this history, it is reasonable to refer to Theorem 4.3 as constituting standard monomial theory for the semigroup $\operatorname{rings} \mathbf{C}\left(\mathbf{Z}^{+}(\geq)\right)$. From this point of view, standard monomial theory for flag algebras would consist of two parts: a flat deformation result, of the sort of Gonciulea-Lakshmibai and successors, and a standard monomial theory for the resulting lattice cone. For a straightforward treatment of standard monomial theory for classical groups built around the GT cone, see $[\mathrm{K}]$.

\section{References}

[BG] C. Benson and L. Grove, Finite Reflection Groups, Bogden \& Quigley, Tarrytown on Hudson, 1971.

[BH] W.Bruns and J.Herzog, Cohen-Macaulay Rings, Cambridge Studies in Advanced Mathematics 39, Cambridge University Press, Cambridge, England, 1993.

[Cal] Ph.Caldero, Toric Degenerations of Schubert Varieites, Trans. Groups 7 (2002), 51-61.

[Chi] R.Chirivi, LS Algebras and Application to Schubert Varieties, Trans. Groups 5 (2000), 245-264.

[DCEB1] C. De Concini, D.Eisenbud, C.Procesi, Young diagrams and determinantial varieties, Inv. Math. 56 (1980), 129-165. 
[DCEB2] C. De Concini, D.Eisenbud, C.Procesi, Hodge Algebras, Asterisque, 91 (1982).

[Fu] W.Fulton, Introduction to Toric Varieties, Ann. Math. Stud. 131, Princeton University Press, Princeton, NJ, 1993.

[GC] I.M.Gelfand and M.L. Cetlin, Finite dimensional representations of the group of unimodular matrices, Doklady Akad. Nauk. SSSR (N.S.) 71 (1950), $825-828$.

[GL] N. Gonciulea and V.Lakshmibal, Degenerations of flag and Schubert Varieties to toric varieties, Trans. Groups 1 (1996), 215-248.

[Hi] T. Hibi, Distributive lattices, affine semigroup rings and algebras with straightening laws, in Commutative Algebra and Combinatorics (Kyoto 1985), Adv. Stud. Pure Math.11, North Holland, Amsterdam, 1987, 93-109.

[Hod] W.Hodge, Some enumerative results in the theory of forms, Proc. Cam. Phi. Soc. 39 (1943), 22-30.

[HP] W.Hodge and D.Pedoe, Methods of Algebraic Geometry II, Cambridge University Press, Cambridge, 1968.

[Ho1] R. Howe, Perspectives on Invariant Theory, in The Schur Lectures (1993), S.Gelbart and I.I. Piatetski-Shapiro, eds. Israel Math. Conf. Proc. 8 (1995), $1-182$.

$[\mathrm{K}]$ S. Kim, Yale Univerity thesis, in preparation.

[Pr] R. Proctor, Young tableaux, Gelfand patterns, and branching rules for classical groups, J. Alg. 164 (1994), 299-360.

[St] R. Stanley, Enumerative Combinatorics, v. 1, Wadsworth \& Brooks/Cole. Monterrey CA, 1986.

Department of Mathematics, Yale University, New Haven CT 06520-8283, USA.

E-mail: roger.howe@yale.edu 